\title{
Anchoring of FtsZ Assemblies to the Chloroplast Envelope: The Role of ARC6
}

\author{
C.B. Johnson***,\#, A. Ravichandran**,\#, A.G. Smith****, S. Vitha*, and A. Holzenburg*,****** \\ * Microscopy \& Imaging Center, Texas A\&M University, College Station, TX 77843-2257 \\ **Department of Biology, Texas A\&M University, College Station, TX 77843-3258 \\ ***Dept. Biochemistry and Biophysics, Texas A\&M University, College Station, TX 77843-2128 \\ ${ }^{\#}$ These authors contributed equally
}

Replication of chloroplast proceeds via binary fission [1] and requires coassembly of FtsZ1 and FtsZ2 proteins to a Z-ring at mid-chloroplast [2]. FtsZ1 and FtsZ2 evolved from a single cyanobacterial ancestor and are structural homologs of tubulin [3]. FtsZ1-FtsZ2 assemblies at the chloroplast division site are stabilized and anchored to the inner chloroplast envelope via interaction of FtsZ2 with a transmembrane protein ARC6 [4]. Plants lacking ARC6 are defective in chloroplast division, contain only one large chloroplast per cell and show abnormal, fragmented FtsZ filaments [5]. As ARC6 serves as an anchor, we hypothesized that FtsZ assemblies will be highly mobile in the absence of the ARC6 anchor. To assess the mobility using in vivo imaging, FtsZ2 was tagged with a green fluorescent protein and expressed in Arabidopsis thaliana wild-type and arc6 mutant plants [2].

Materials and Methods

Leaves from Arabidopsis thaliana wild-type and arc6 mutant plants expressing AtFtsZ2-1 Cterminally tagged with GFP [2] were mounted in perfluorodecalin [6] and viewed using a Olympus FV1000 confocal microscope (60x/1.2 water immersion objective) or a wide-field Zeiss Axiophot microscope (63x/1.4 oil immersions objective) with appropriate fluorescence filters and a CoolSnap cf CCD camera (Photometrics). Time lapse image series of 200 frames were recorded with frame intervals between 200 and $500 \mathrm{~ms}$ (wide-field) or $580 \mathrm{~ms}$ (confocal) and analyzed using ImageJ software (http://rsb. info.nih.gov/ij) and the SpotTracker plugin [7].

Results

Similar to prior results of immunofluorescence localization [8], two different types of FtsZ assemblies were observed in the arc 6 genetic background: longer filaments as well as puncta. While the longer filaments remained essentially anchored and exhibited only some flexing, bending and occasionally also flailing of the ends, the puncta either oscillated around a fixed location or exhibited longer-range directional movement (Fig. 1A). A given punctum may transition from one type of movement to the other. As these observations were made in the absence of ARC6, the observed partial restriction in movement speaks to a second anchoring mechanism, possibly via the PARC6 membrane-anchored protein that interacts with the FtsZ1 protein [9]. The fact that longer range movements have also been observed could be due to limiting availability of anchoring sites, i.e. the number of anchors is not sufficient to immobilize the FtsZ assembly in these plants. The latter possibility is also corroborated by observations with wild-type plants overexpressing the AtFtsZ2-1GFP transgene. In these plants the stoichiometry of FtsZ and the anchoring proteins is altered resulting in a large number of FtsZ mini-rings housed in enlarged chloroplasts. These mini-rings (Fig. 1B) also exhibit oscillatory and longer-range movements consistent with an insufficient number of anchors to immobilize the FtsZ assemblies [10]. 
References

[1] K.W. Osteryoung and J. Nunnari, Science, 302 (2003) 1698.

[2] S. Vitha et al., J. Cell Biol., 153 (2001) 111.

[3] S.Y. Miyagishima et al., J. Mol. Evol., 58 (2004) 291.

[4] J.M. Glynn et al., Plant Cell, 20 (2008) 2460.

[5] S. Vitha et al., Plant Cell, 15 (2003) 1918.

[6] G.R. Littlejohn et al., New Phytol, 186 (2010) 1018.

[7] D. Sage et al., IEEE Trans Image Process, 14 (2005) 1372.

[8] S. Vitha et al., Plant Cell, 15 (2003) 1918.

[9] J.M. Glynn et al., Plant J., 59 (2009) 700.

[10] The Olympus FV1000 confocal microscope acquisition was supported by the Office of the Vice President for Research at Texas A\&M University.
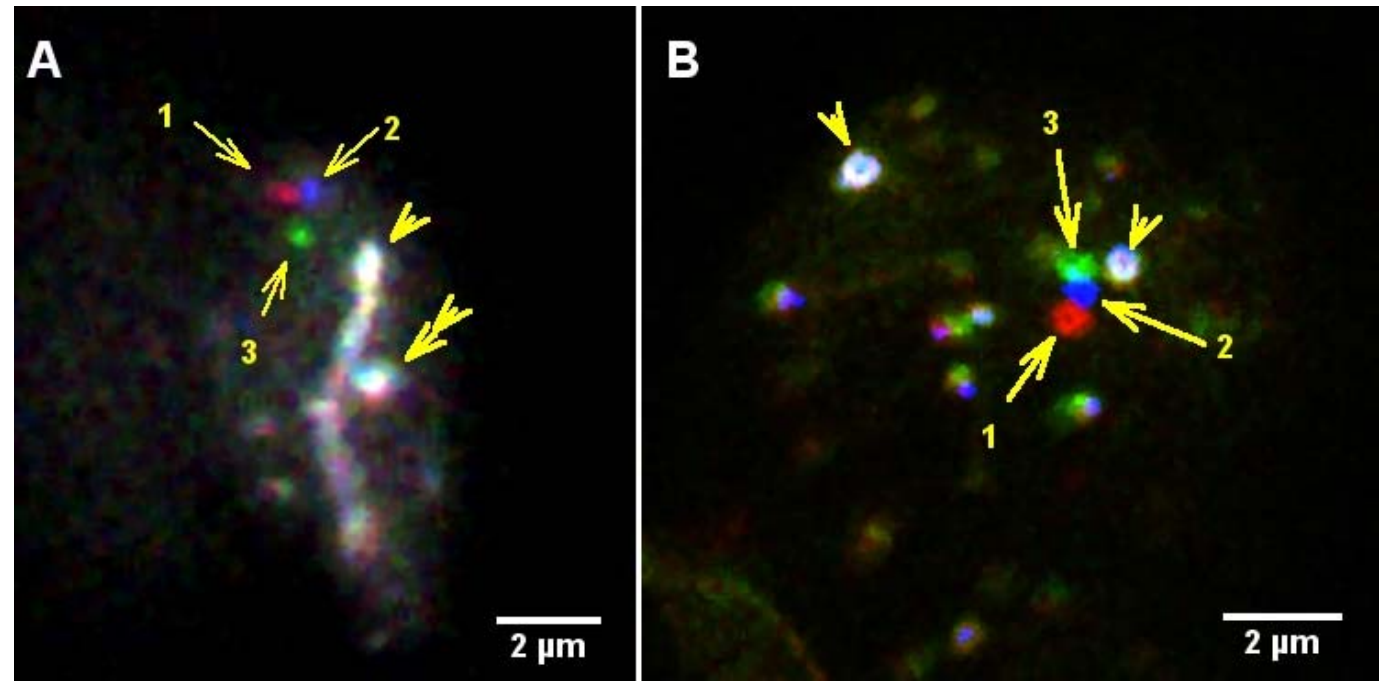

FIG. 1. Color overlay of three consecutive frames from time-lapse microscopy. (A) Confocal imaging of FtsZ2-1-GFP in an arc6 mutant background. The relatively long FtsZ filament (arrowhead) and one punctate FtsZ assembly (double arrowhead) are stationary and shown in white. The positions of a mobile punctum in frames 1 (red), 2 (blue) and 3 (green) are highlighted by arrows. (B) Wide-field imaging of FtsZ2-1-GFP in a wild-type background. Stationary mini-rings are again marked by arrowheads. The positions of a mobile mini-ring in frames 1 (red), 2 (blue) and 3 (green) are highlighted by arrows. 